\title{
LA EVALUACIÓN Y EL TRABAJO COLABORATIVO COMO APORTE AL PROCESO EDUCATIVO
}

The review and collaborative work as a contribution to educational process

A avaliação e o trabalho colaborativo, como aporte ao processo educativo

\section{Sandra Carolina Sánchez Miñán (1)}

(1) Universidad de Valparaíso, Chile. Fono: 2603831-95969972. Correo electrónico: sandra.sanchez@uv.cl

\section{Resumen}

Se aborda una temática que está orientada a trabajar colaborativamente, aceptando y respetando a cada persona, ya que todos van aportando al proceso evaluativo y éste se vuelve más significativo cuando considera la opinión, información y visión de quienes participan de este espacio social y cultural, lo que además se relaciona con las diversas realidades chilenas.

Palabras clave: Evaluación, evaluación diferenciada, trabajo colaborativo, proceso educativo.

\section{Summary}

A theme that is oriented to work collaboratively, accepting and respecting each person, since all are contributing to the evaluation process and it becomes more significant when it is consider the opinion, information and vision of those who participate in the social and cultural space, which also relates to the various Chilean realities.

Keywords: review, differentiated assessment, collaborative work, educational process.

\section{Resumo}

Aborda-se uma temática que está orientada a trabalhar em forma colaborativa, aceitando e respeitando a cada pessoa, já que todos vão aportando ao processo avaliativo e este torna-se mais significativo quando considera a opinião, informação e visão dos que participam de este espaço social e cultural, o que ademais se relaciona com as diversas realidades chilenas. 
Palavras chaves: Avaliação, avaliação diferenciada, trabalho colaborativo, processo educativo.

\section{La evaluación y el trabajo colaborativo desde la diversidad de saberes y experiencias}

En los contextos socioeducativos se busca que los componentes básicos del desarrollo curricular, tales como la planificación, el espacio, tiempo, evaluación, organización y conformación de comunidades educativas, se lleven a cabo de forma coherente y adecuada para apoyar el logro de aprendizajes de niños y niñas.

Uno de esos componentes corresponde a la evaluación, la que se entiende “...como un proceso permanente y sistemático, mediante el cual se obtiene y analiza información relevante sobre todo el proceso de enseñanza-aprendizaje, para formular un juicio valorativo que permita tomar decisiones adecuadas que retroalimenten y mejoren el proceso educativo en sus diferentes dimensiones." (Bases Curriculares de la Educación Parvularia, 2004: 107), lo que se torna un elemento trascendental en todo espacio pedagógico, cuando está orientado a lograr una educación de calidad y mejorar prácticas de enseñanza para que los estudiantes construyan conocimientos de forma significativa.

Es así que surge la necesidad de referirse a la evaluación auténtica, cuyo propósito es mejorar los procesos de enseñanza y aprendizaje generados en la interacción de los educadores/as con los estudiantes y a la vez orientar a la familia sobre los avances que presentan sus hijos/as, enfocándose en sus habilidades, competencias e intereses. (Condemarín y Medina, 2010)

Desde esta perspectiva, la evaluación se convierte en un aporte para los diversos profesionales, ya que tienen la oportunidad de autoevaluar su desempeño, analizar y reflexionar de manera crítica, lo cual va a permitirles volver a mirar sus acciones en relación a la mediación e interacción con los niños y niñas, las familias y los profesionales que son parte de la escuela y de esta forma mejorar sus prácticas pedagógicas y el trabajo que desarrollan tanto en el aula como fuera de ella. 
Respecto a la evaluación y todo lo que implica el trabajo pedagógico desarrollado en el aula, es que se hace necesario que exista un enfoque orientado en la educación para la diversidad, que considere a cada estudiante con sus características personales, valores, creencias, costumbres, tradiciones, necesidades, intereses, diversas historias de vida y necesidades educativas especiales que reflejan la interacción de diferentes personas y culturas (Araneda et al. 2006).

Si bien se convierte en un desafío para quienes participan de la educación, es importante "avanzar hacia el desarrollo de comunidades abiertas y respetuosas de la diversidad, capaces de proporcionar una educación común a todos los estudiantes y a la vez diversificada..." (Lucchini, 2009: 193) que acepten y valoren a las personas y posean una mirada heterogénea de lo que implica educar.

Sobre esta base, el objetivo de la evaluación diferenciada, apunta a facilitar el desarrollo de niños y niñas que presentan dificultades de aprendizajes y lograr que puedan incorporarse al grupo de estudiantes y junto con ello posibilitar los procesos educativos, además “... es deseable modelos evaluativos de base criterial y que refleje logros o avances realizados por el niño(a) sobre sí mismo..." (Donoso et al. 2006: 179), sin establecer comparaciones y que permita obtener información al inicio, desarrollo y término de los procesos de enseñanza y aprendizaje.

Para que este proceso evaluativo se desarrolle a cabalidad, es fundamental la participación de los equipos de aula (educadoras/es de párvulos y educadoras/es diferenciales) y de los equipos de apoyo (fonoaudiólogos/as, psicólogos/as, entre otros), de la familia y de los propios niños y niñas, ya que cada uno aporta con información relevante para que los educadores/as preparen y organicen la enseñanza de una mejor manera.

Ello implica realizar un trabajo en el que se da énfasis a la colaboración, es decir, “...cuando vemos a varias personas coordinando sus haceres y emociones en alguna tarea particular en el placer de hacerlo, sin esperar otro beneficio que ese placer" (Dávila y Maturana, 2008: 386), que se vincula con el desarrollo pleno e integral de niños y niñas, basado en espacios de respeto mutuo y de construcción significativa. 
Por lo tanto los educadores/as necesitan trabajar de forma colaborativa con "...los psicólogos, los trabajadores sociales y los profesionales de la salud, de manera que puedan utilizar sus evaluaciones especializadas para propósitos educativos." (Oficina Regional de Educación de la UNESCO, 2004: 60), lo que quiere decir que se requiere una comunicación fluida y constante. Esto genera aportes desde distintas visiones, no solo de los profesionales sino que también de la familia que acompaña este proceso educativo y que otorga apoyos a sus hijos/as, donde cada uno observa y se relaciona con los niños y niñas en diferentes contextos sociales, por ello la importancia de mantener un diálogo permanente.

Todo esto implica una gran responsabilidad y compromiso de los profesionales, ya que requiere “...la puesta en marcha de un conjunto de acciones planificadas y coordinadas para conseguir que "todos/as" los estudiantes participen y aprendan en un contexto que comprende sus dificultades, valora sus diferencias y provee los apoyos necesarios, en un clima de confianza y respeto.” (Orientaciones Técnico-Pedagógicas para la Evaluación Diagnóstica Integral, 2010: 3), en espacios educativos en los que se hace necesario utilizar procedimientos e instrumentos de evaluación acordes a las características que presenta cada niño y niña. Lo que demanda la “...creación de una comunidad escolar segura, acogedora, colaboradora y estimulante, en la que cada uno es valorado, lo cual es la base fundamental primordial para que todo el alumnado tenga mayores niveles de logro." (Ainscow y Booth, 2000: 18), ello se va construyendo con la apertura y disposición de todas las personas que participan de los contextos sociales y educativos, cuando se logra, se benefician las personas y se enriquece el proceso educativo en sí.

En cuanto a la evaluación y a los procesos educativos, es necesario destacar que participan los niños y niñas, quienes son los protagonistas de sus aprendizajes, poseen intereses, necesidades, ritmos y estilos propios para construir sus conocimientos. Algunos de ellos pueden presentar necesidades educativas especiales transitorias o permanentes, por lo que necesitan otro tipo de apoyos para lograr avances en el ámbito educativo y es aquí donde se vuelve relevante el trabajo de las/os educadoras/es como profesionales que planifican y organizan la enseñanza, evalúan los avances de los niños y niñas, toman decisiones pedagógicas, entre otras funciones que desarrollan y que se complementan con 
el trabajo que realizan los profesionales de la salud, los cuales apoyan desde sus conocimientos, otorgando antecedentes relevantes, evaluando desde otras perspectivas relacionadas con su área e incluyendo a educadores/as y familias, éstas últimas constituyen el núcleo básico en el que se desenvuelven y se identifican los niños y niñas, ya que generan los primeros vínculos y lazos afectivos.

Tanto educadoras/es de párvulos y educadoras/es diferenciales necesitan trabajar en conjunto, puesto que “...es esencial para brindar una oferta pedagógica de calidad y ajustada a las necesidades educativas especiales de cada niño y niña que requiere de adecuaciones en el quehacer educativo." (Araneda et al. 2006: 12) y que demandan una mirada de ambos profesionales, que otorgue un apoyo integral a los estudiantes. Lo que genera aunar criterios pedagógicos, adecuar, diseñar, implementar y evaluar los componentes básicos del desarrollo curricular, de manera sistemática y permanente, así como también cuando sea conveniente, ya que se busca potenciar las habilidades de los niños y niñas, optimizar las prácticas de enseñanza y preparar los ambientes de aprendizaje.

\section{Una mirada desde la realidad}

Al abordar la evaluación se requiere explicar que ésta se enmarca en “...una cuestión de todos y para todos. No debe ser una práctica conducente al individualismo y a la competitividad." (Santos Guerra, 2007: 18), ya que cada uno avanza de acuerdo a sus ritmos particulares, a sus vivencias y experiencias personales. Este proceso de evaluación se desarrolla en contextos educativos, los cuales se convierten en espacios socioculturales, que son el reflejo de lo que ocurre en la sociedad, ambientes que se van construyendo y creando a partir de las interacciones de las diversas personas que confluyen en un mismo entorno.

Espacios en los que "muchas veces los niños y niñas quedan expuestos al desconocimiento de su diversidad por parte de su entorno educativo, puesto que la tendencia común es a homogeneizar o asimilar habilidades...” (Araneda et al. 2006: 16-17), dejando de lado la identidad social, cultural y las características de los estudiantes y de cada persona que conforma estos contextos. 
Estos espacios socioculturales, correspondientes a las escuelas, son ambientes constituidos por seres humanos que se interrelacionan y conviven entre sí, por ende cada realidad es diversa, pero los objetivos son comunes y éstos apuntan a lograr una educación de calidad, con procesos de enseñanza y aprendizaje acordes a las necesidades e intereses de los niños y niñas. Todo ello “...demanda nuevas exigencias y competencias al profesorado y, por tanto, la adopción de un conjunto de medidas de apoyo que ayuden a las escuelas a enfrentar con éxito los cambios que exige la atención a la diversidad" (Lucchini, 2009: 194), labor que implica un gran desafío para quienes se encuentran participando, haciéndose necesario realizar un trabajo colaborativo entre todos, de forma que sea una experiencia compartida.

Avanzar en esta labor requiere de un currículum flexible que se adapte a los ritmos, estilos, habilidades y necesidades educativas especiales que presentan algunos niños y niñas y que demandan recursos, ajustes y estrategias pedagógicas apropiadas. Es primordial que no solo sean teorías o que permanezcan en el discurso, sino que sean llevadas a la práctica, ya que con frecuencia se observa en las realidades educativas chilenas que se da énfasis a enfoques homogeneizadores, en donde no existe una orientación real al respecto.

Si bien "el objetivo de la evaluación es hacer posible que los docentes y escuelas den respuesta a una amplia diversidad de estudiantes." (Oficina Regional de Educación de la UNESCO, 2004: 59), con frecuencia se vuelve una tarea compleja de llevar a cabo en la educación chilena, por factores que repercuten entre sí, como es el caso de las funciones y responsabilidades que asumen los profesionales, horarios extensos de trabajo, gran cantidad de estudiantes en el aula, variadas actividades, tales como, reuniones de apoderados, consejos de profesores, evaluaciones, informes pedagógicos, así como también las características personales y el ambiente laboral que se genera debido a dichas condiciones.

Para lograr un trabajo en conjunto es fundamental realizar una labor colaborativa, ya que "...es la principal herramienta para mejorar la calidad de los aprendizaje de todos/as los estudiantes, especialmente de los que presentan NEE." (Orientaciones para la Implementación del Decreto $\mathrm{N}^{\mathrm{o}} 170$ en Programas de Integración Escolar, 2010: 13), de 
manera que los equipos de aula y los equipos de apoyo, las familias y los propios niños y niñas, puedan ir aportando desde distintas visiones y al mismo tiempo aunando criterios que orienten el camino a seguir en la educación de los niños y niñas con necesidades educativas especiales o que requieran de determinados apoyos en su proceso de aprendizaje.

Algunos aspectos a considerar para todos esos niños y niñas, corresponden al desarrollo socioemocional, es decir, necesidades biológicas y emocionales, promoviendo contextos basados en interacciones positivas, para que se sientan seguros, acogidos, valorados y respetados, procurando una buena comunicación, propiciando el respeto de espacios de sana convivencia, mediando adecuadamente los aprendizajes, según las características de cada estudiante, ya que aquellos que presenten necesidades educativas especiales, requerirán de otros apoyos pedagógicos. (Araneda et al. 2006)

Por otra parte, el Decreto $\mathrm{N}^{\mathrm{o}} 170$ establece que “...los equipos de profesionales, deben utilizar prioritariamente, instrumentos, pruebas o test con normas nacionales" (Decreto $\mathrm{N}^{\mathrm{o}}$ 170, 2009: 2), para recoger información y posteriormente definir los aprendizajes a intencionar, sin embargo, es necesario que se pueda complementar con otros instrumentos de evaluación, no solo cuantitativos, sino además cualitativos, que consideren los progresos de los niños y niñas. La escuela y por ende los equipos de aula, también pueden elaborar sus propias pautas y protocolos de evaluación para los estudiantes que presenten necesidades educativas especiales y también para el grupo, de tal forma que se enriquezca el proceso evaluativo y principalmente que las evaluaciones sean significativas considerando a cada uno/a.

Es una tarea que implica un desafío para el educador/a al momento de organizar y coordinar estas funciones, en un tiempo que necesariamente se torna flexible, ya que en ocasiones emergen situaciones, cuando se trata de realizar un trabajo en equipo, que de alguna manera determinan las acciones que continuarán desarrollándose. Asimismo ocurre con las actividades y responsabilidades de los equipos de aula, ya que gran parte del tiempo se desempeñan en este espacio y son breves los períodos con los que cuentan para otros compromisos, relacionados con la preparación para la enseñanza y el aprendizaje y con 
aspectos evaluativos, debiendo muchas veces cumplir con algunos de ellos en contextos externos a la escuela.

Junto con ello, el Decreto $\mathrm{N}^{0} 170$ expone que los programas de integración escolar deberán construir una planificación, con el tiempo que los profesionales consignarán para actividades como:

- Apoyo a estudiantes en el aula común.

- Planificaciones, evaluaciones, preparación de materiales educativos, entre otras, en colaboración con los educadores/as de educación regular.

- Trabajo con los niños y niñas, de manera individual o grupal (pequeños grupos), con la familia, otros profesionales y equipo directivo del centro educativo. (Decreto $\mathrm{N}^{\mathrm{o}}$ 170, 2009).

Todo esto constituye instancias para lograr un trabajo colaborativo. Lo importante es cautelar dentro de cada establecimiento educacional que se pueda realizar, en espacios pertinentes y en horarios acordados para que sea una labor conjunta y real.

Como se ha expresado, la evaluación repercute en diversos elementos del proceso de enseñanza y aprendizaje y busca mejorar las prácticas educativas, en consecuencia, para que esto "...se produzca es necesario que la disposición de los que intervienen en ella sea abierta y comprometida. Si no existe actitud autocrítica y apertura a la opinión externa es muy difícil que cambie algo profundamente.” (Santos Guerra, 2007: 51), ya que se generarán transformaciones sin un sentido, lo que implica que escasamente se pueda optimizar la enseñanza y aprendizaje y los procesos evaluativos.

Otra característica a analizar corresponde a la cantidad de niños y niñas presentes en la sala de actividades, debido a que los educadores tienen que adaptar las metodologías de enseñanza considerando grupos numerosos, en los que cada uno posee características personales, intereses, necesidades y habilidades y en donde se busca el logro de aprendizajes para todos, es allí cuando los equipos de aula utilizan diversas estrategias pedagógicas, en que los estudiantes participen colaborativamente, aportando unos a otros en las situaciones educativas y en cuyos espacios los educadores/as cumplen un rol 
mediador, que orienta, aclara y explica, para que niños y niñas construyan sus conocimientos. Todo ello se convierte en instancias de evaluación y a la vez de retroalimentación, para quienes forman parte del proceso.

En relación a los contextos y ambientes de aprendizaje, es relevante "cautelar que los materiales estén adecuadamente organizados y seleccionados de acuerdo a las características de los niños y de los aprendizajes esperados.” (Bases Curriculares de la Educación Parvularia, 2004: 102), ya que al preparar experiencias educativas, es primordial que los materiales o recursos pedagógicos se conviertan en un aporte y que consideren las características del grupo y las necesidades educativas especiales que presenten los niños y niñas.

Sobre esta base es que "la mayor dificultad que tenemos para comprender nuestro vivir está en que no nos hacemos cargo de que el mundo que vivimos no preexiste a nuestro vivirlo sino que surge con nosotros en nuestro vivir y convivir aunque lo vivamos como si preexistiese a nuestro distinguirlo" (Dávila y Maturana, 2008: 207), lo que significa que vivir es una construcción constante que implica aceptarse a sí mismos y a los demás con características propias, respetando formas de vida que son personales y se constituyen en la convivencia constante y recurrente que abre paso a la valoración y apertura, al momento de plantear opiniones, argumentos y puntos de vista, dentro de contextos educativos que demandan una educación para la diversidad. Para que exista coherencia es fundamental continuar con este desafío desde los adultos que conforman ese espacio, para que trascienda a los niños y niñas y a todos quienes coexisten en la comunidad educativa.

\section{Referencias bibliográficas}

Ainscow, M y Booth, T. (2000). Índice de Inclusión. Desarrollando el aprendizaje y la participación en las escuelas. Bristol.

Araneda, P. Calisto, P. Cortéz, N. González, F. Miranda, M. Muñoz, C. y Negrotti, C. (2006). Guía Orientaciones Pedagógicas. Para la Atención a la Diversidad de Niños y 
Niñas con Necesidades Educativas Especiales en la Educación Parvularia. Santiago de Chile: Atenas Ltda.

Ballester, M. Batalloso, J. Calatayud, M. Córdoba, I. Diego, J. Fons, M. Giner, T. Jorba, J. Mir, B. Moreno, I. Otero, L. Parcerisa, A. Pigrau, T. Pitaluga, I. Pujol, M. Quinquer, D. Quintana, H. Sanmartí, N. Sbert, C. Sbert, M. y Weissman, H. (2000). Evaluación como ayuda al aprendizaje. Barcelona: Graó, de IRIF, SL.

Condemarín, M. y Medina, A. (2010). Evaluación auténtica de los aprendizajes. Un medio para mejorar las competencias en lenguaje y comunicación. Santiago de Chile: Andrés Bello.

Dávila, X. y Maturana, H. (2008). Habitar Humano. En seis ensayos de BiologíaCultural. Santiago de Chile: Comunicaciones Noreste Ltda.

Donoso, M. Durán, R. y Muñoz, E. (2006). Atención a la Diversidad en el Aula Común. Santiago de Chile: Libart.

Lucchini, G. (2009). Niños con Necesidades Educativas Especiales. Cómo enfrentar el trabajo en el aula. Santiago de Chile: Universidad Católica de Chile.

Ministerio de Educación. (2004). Bases Curriculares de la Educación Parvularia. Santiago de Chile: Valente.

Ministerio de Educación. (2009). Decreto $N^{o} 170$. Santiago de Chile. 
Ministerio de Educación. (2010). Orientaciones Técnico-Pedagógicas para la Evaluación Diagnóstica Integral. Santiago de Chile.

Ministerio de Educación. (2010). Orientaciones para la Implementación del Decreto $N^{o}$ 170 en Programas de Integración Escolar. Chile.

Oficina Regional de Educación de la UNESCO. (2004). Temario Abierto sobre Educación Inclusiva. Materiales de Apoyo para Responsables de Políticas Educativas. Santiago de Chile: Archivos Industriales y Promocionales Ltda.

Santos, M. (2003). Dime cómo evalúas y te diré qué tipo de profesional y de persona eres. Enfoques Educacionales, Volumen No 5, 69-80.

Santos, M. (2007). La evaluación como aprendizaje. Madrid: NARCEA, S.A. de Ediciones. 